\title{
Highly Selective and Sensitive Detection of Methylbenzenes via Concurrent Control of the Gas Reforming and Filtering in Catalytic Oxide Overlayer.
}

\author{
Jun-Sik Kim ${ }^{1}$, Hyun-Mook Jeong ${ }^{1}$, Jae-Hyeok Kim ${ }^{1}$, Bo-Young Kim ${ }^{1}$, Ho Won Jang ${ }^{2}$, Jong-Heun Lee ${ }^{1}$ \\ ${ }^{1}$ Department of Materials Science and Engineering, Korea University, Seoul 02841, Republic of Korea \\ ${ }^{2}$ Department of Materials Science and Engineering, Research Institute of Advanced Materials, Seoul \\ National University, Seoul 08826, Republic of Korea \\ jongheun@korea.ac.kr
}

\begin{abstract}
$\mathrm{A} \mathrm{Co}_{3} \mathrm{O}_{4}$ gas sensor coated with catalytic oxide overlayer was fabricated by screen printing of $\mathrm{Co}_{3} \mathrm{O}_{4}$ yolk-shell spheres on the substrate and subsequent deposition of $\mathrm{SnO}_{2}$ or $\mathrm{TiO}_{2}$ catalytic overlayer by e-beam evaporation. $\mathrm{Co}_{3} \mathrm{O}_{4}$ gas sensors with $\mathrm{TiO}_{2}$ and $\mathrm{SnO}_{2}$ overlayer (thickness: $5 \mathrm{~nm}$ ) showed high responses (resistance ratios) to $5 \mathrm{ppm}$ xylene $(14.5,28.8)$ and $5 \mathrm{ppm}$ toluene $(11.7,16.2)$ at $250{ }^{\circ} \mathrm{C}$ and exhibited excellent selectivity against other interfering gases such as ethanol, $\mathrm{HCHO}, \mathrm{CO}$, and benzene. In contrast, the pure $\mathrm{CO}_{3} \mathrm{O}_{4}$ gas sensor did not show high selectivity toward any specific gas. The excellent selectivity to methylbenzenes was attributed to catalytic reforming of less reactive methylbenzene into more reactive and smaller species and catalytic oxidation of reactive interference gases such as ethanol and $\mathrm{HCHO}$ at catalytic overlayer. These concurrent tuning of the gas reforming and oxidative filtering processes using a nanoscale catalytic oxide overlayer provides a new, general, and effective method for fabricating highly selective and sensitive metal oxide semiconductor gas sensors.
\end{abstract}

Key words: $\mathrm{Co}_{3} \mathrm{O}_{4}$ gas sensor, methylbenzenes, catalytic overlayer, gas reforming, gas filtering 